\title{
CORRECTIONS
}

\section{Innovation in healthcare: Finalists reflect a wealth of potential}

In her article describing the three innovation finalists in the BMJ Group awards (BMJ 2011;342:d2087, doi:10.1136/bmj.d2087), Luisa Dillner describes Tricia Lewis as the project manager of the "bubbalicious" website, whereas she is, in fact, the website's referee.

Cite this as: $B M J$ 2011;342:d2377 